\title{
MEGALENCEPHALY: A REPORT OF TWO CASES
}

BY

\author{
JOHN APLEY AND MICHAEL SYMONS
}

(From the Departments of Medicine and Pathology, University of Bristol)

While there can be little doubt that megalencephaly is a rare condition, the degree of rarity is possibly exaggerated. In both the cases described below, seen within a week of each other, a mistaken diagnosis of hydrocephalus was made clinically, and without autopsy would have remained uncorrected. It seems quite probable that some cases of megalencephaly are erroneously labelled hydrocephalus, and with this diagnosis post-mortem examination is not uncommonly omitted.

Apart from the value of collating evidence in an uncommon condition these cases may throw some light on the mode of death in association with raised intracranial tension.

\section{Case Histories}

Janet G. She was an only child, with no relevant family history, and was born one month before the expected date, was slightly cyanosed at birth, and weighed $5 \mathrm{lb} .4 \frac{1}{2} \mathrm{oz}$. No note was made as to any cranial abnormality. From the first she did not suck well, and the gain in weight was unsatisfactory. At the age of six and a half months she was not yet attempting to sit up or grasp objects, and the head circumference was $19 \cdot 5$ in. (normal $17 \cdot 3$ in.). At nine months the head was described as 'hydrocephalic' and measured 20 in. in circumference. At one year she could not speak at all, moved her limbs very little, and was considered to be mentally defective.

Present illness. At the age of one year she became acutely ill and for two days cried frequently, as if in pain, and did not sleep well. After the second day she vomited once. She was found by her doctor to have a raised temperature, head retraction, and a positive Kernig's sign.

On admission to hospital the temperature was $101^{\circ} \mathrm{F}$., and the pulse and respiration rate were respectively 140 and 30 per minute. The head appeared large. The child gave the impression of not seeing properly, though she blinked at objects moved towards the eyes. The anterior fontanelle was widely patent and the forehead prominent. There was frequent twitching of the face, markedly on the right side. The child did not move her head or attempt to sit up, but the limbs moved and she could swallow. Kernig's sign was now indefinite, the deep reflexes were normal, and plantar responses flexor. The tonsils were enlarged and red, but routine clinical examination was otherwise unproductive.

Lumbar puncture was performed next day, and $10 \mathrm{c.cm}$. of fluid removed. The initial pressure of
$165 \mathrm{~mm}$. of water rose to $190 \mathrm{~mm}$. with the Queckenstedt manoeuvre, and slowly fell again on releasing the pressure. Shortly after removal of the fluid, which was found to be normal, convulsive movements of face, arms, and legs occurred.

Later in the day the temperature, pulse, and respiration began to rise from $104^{\circ} \mathrm{F}$. and 140 and 40 per minute, and by the next day had reached respectively $109 \cdot 2^{\circ} \mathrm{F}$., 170 , and 50 , when the child became collapsed and died.

POST-MORTEM FINDINGS. The body was well nourished and the only apparent abnormality was the large head, which measured 20 in. in its greatest circumference. The head was normal in shape and did not have the bulging appearance usually seen in hydrocephalus.

There was slight tonsillar enlargement. In the remainder of the body, apart from the skull and brain, no abnormalities were found. The sutures of the calvarium appeared firmly united but the bone was thinner than normal. The anterior fontanelle was widely open but not bulging; the other fontanelles were closed. The meninges appeared normal. The cerebral convolutions were not flattened, though there was a slight compression cone on the under surface of the cerebellum. The whole brain weighed $1,450 \mathrm{~g}$. (normal for this age approximately $975 \mathrm{~g}$. See Plate VIII, figs. 1 and 3). The increase in size affected all areas of the brain proportionately. The right cerebral hemisphere was sectioned at once and appeared microscopically normal. The ratio of white to grey matter was within the normal range, and the ventricular system was not dilated. The remainder of the brain was kept for histological study by Dr. R. M. Norman, who reported that he could find no apparent abnormalities in routine blocks from different parts of the brain. The spinal cord was not examined.

Autopsy diagnosis : megalencephaly; tonsillitis; pressure cone of cerebellum.

Linda B. Of this child's two siblings, one was premature. An uncle is said to suffer from attacks of petit mal.

PERSONAL history. Delivered normally at full term, she weighed $7 \mathrm{lb}$. $9 \frac{1}{2}$ ozs. at birth. The mother's age at delivery was twenty-three years, and the child was described as ' a healthy female baby.' At birth the head measurements were recorded as follows : circumference 13.5 in. ; S.O.B. 3.75 in.; S.O.F. 4 in. ; occipito-frontal 4.5 in. ; M.V. 5.25 in. ; S.M.B. 3.75 in. ; B.P. 3.75 in. ; B.T. 3.5 in. These figures are normal.

The child's early development showed no significant deviations from normal, but she is said to have been 'chesty' since the age of two months, 
and at ten months had 'influenza' with running at the eyes and nose for a week. Subsequently she gained weight only poorly throughout the winter. One week before admission to hospital, at the age of fifteen months, she was given her first antidiphtheria inoculation.

Present ILlness. At this time the child was described as 'off colour.' Two days later a cough developed, and in a further two days she began to vomit most of her feeds. On the morning of admission to hospital a fit occurred, during which it was said that the eyes rolled up, the child was limp, then stiff, and then went ' black.' She came to when put into a hot bath, but a similar, rather milder, attack occurred a few hours later.

On admission the child looked grey and ill. She was dyspnoeic and grunted with each respiration. The throat appeared mildly inflamed. In the chest scattered rhonchi were audible, and there was doubtfully impaired resonance at the left base. The pulse rate was rapid, but the heart was considered to be normal. No abnormalities were detected in the abdomen. The anterior fontanelle was still widely patent. Apart from slight hypotonia there was no evidence of central nervous system involvement. The temperature was $100^{\circ} \mathrm{F}$., the pulse 160, and the respiration 44 . Sulphadiazine was prescribed. Vomiting occurred once on the day of admission.

Suddenly, on the next day, breathing stopped and the child became cyanosed and unconscious. All four limbs were then flaccid and the plantar responses extensor. In a few minutes, when breathing started, moist sounds were heard throughout the lungs. After half an hour the limbs became hypertonic. Consciousness was not fully recovered two and a half hours after this incident, when again breathing stopped for a few minutes. Marked cyanosis developed and the pulse became impalpable. At this time the child was flaccid, the pupils were small, and the plantar responses were still extensor. The temperature was $105^{\circ} \mathrm{F}$., the pulse 200 , and the respiration 40 . Two and a half hours later a similar incident occurred, and lobeline was injected.

The presence of lesions in the lungs and central nervous system suggested a clinical diagnosis of miliary tuberculosis with meningitis, but this was disproved by cerebrospinal fluid examination. With therecent fatality (case 1) in mind, only a small amount of fluid was withdrawn, insufficient to permit measurement of the pressure; the fluid did not flow at an excessive rate and was clear. The Quecken. stedt test was normal. Cerebrospinal fluid examination showed a clear colourless fluid containing one lymphocyte per c.mm., and protein and chlorides were respectively $35 \mathrm{mg}$. and $760 \mathrm{mg}$. per $100 \mathrm{c.cm}$.

Intramuscular penicillin injections were commenced. During the day the pupils gradually became pin-point in size, the eyes converged, and tremors developed in the eyelids and right arm. Twenty-one hours after the onset of the first attack in hospital breathing ceased again. Oxygen was given and artificial respiration applied, and breathing was resumed. Twelve hours later the temperature was $105.6 \mathrm{~F}$., the pulse rate was too rapid to be counted, and the respiration rate had risen to 60 per minute. Soon after the child died.

POST-MORTEM FINDINGS. The only unusual feature was the large head, which measured 20 in. in its greatest circumference (normal.18.5 in.).

The only abnormalities in the viscera were a patchy bronchopneumonia affecting both lungs, and a congenital stenosis of the right pelvi-ureteric junction with dilatation of the ureter below this in its middle third. The sutures of the cranial vault were united and the bone was somewhat thinned. The anterior fontanelle was still widely open; the other fontanelles were closed. The meninges were healthy, but all the intracranial venous sinuses were markedly distended and congested.

The cerebral convolutions showed some flattening (Plate VIII, fig. 2) and the surface of the brain was dry. The whole brain weighed $1,770 \mathrm{~g}$. (normal for this age approximately 960 g.). There was a proportionate enlargement of the entire brain, which showed no gross abnormalities. The ratio of white to grey matter was normal and the ventricular system was not dilated. Dr. Norman examined the brain histologically and reported that he could find no abnormality. The spinal cord was not examined.

Autopsy diagnosis : megalencephaly; congenital stenosis of the right pelvi-ureteric junction ; bronchopneumonia.

\section{Discussion}

Definition of megalencephaly. The term megalencephaly was first used by Morley Fletcher (1900). As employed by Kinnier Wilson (1933) it is restricted to cases in which there is proportionate enlargement of the whole brain associated with some mental or nervous aberration. This usage eliminates those instances described by other authors in which the enlargement is disproportionate.

Pathology. Cases showing enlargement of the brain may be divided into two groups. In the first, into which our cases fall, there is a proportionate enlargement of the whole brain and, in the gross, all areas appear to be equally affected. The majority of cases are of this type. In the other, smaller group the enlargement of the brain is disproportionate and one part of the brain enlarges, as it were, at the expense of another; thus the cerebral hemispheres may be grossly enlarged and the cerebellum very small, or the ratio of white to grey matter may be abnormal. The histological picture is very variable in both groups. Appearances may be normal, as in our cases; or, at the other extreme, the enlargement is found to be due to a diffuse overgrowth of neuroglial tissue, the so-called gliomatosis. Between these examples are found many varying abnormalities of the brain tissue. There may be a slightly disproportionate increase of one element with normal appearances of the 
tissues themselves, or, as in the case described by Kinnier Wilson (1933), the nerve cells may show abnormal staining reactions and morphology.

Although both the cases described here fulfil the essential criteria of megalencephaly, no histological changes could be found. It is unfortunate that we did not examine the spinal cords, as there does not appear to be any reference in the literature to the possibility of an associated enlargement of this part of the central nevvous system.

Clinical aspects. Megalencephaly or hydrocephalus may be present at birth or develop later, so the time of onset affords no help in differentiation. In both conditions mental deficiency is also common. Meticulous clinical examination should, however, provide some guidance if megalencephaly is borne in mind. In this condition the maximum width of the head is at a level lower than in hydrocephalus (Stewart, 1936-37), in which it lies above the superciliary margin. Encephalography has enabled the diagnosis to be made during life in one recorded case (Brain, 1936).

Recorded cases of megalencephaly are so few that it remains unprofitable to draw conclusions as to prognosis. It seems likely that, in its milder forms, megalencephaly may be benign if a fatal issue is not brought about, as in the cases described, apparently in association with an acute infection.

Cause of death. In some of the recorded cases no details of the cause of death are given, but in many of the remainder death was comparatively sudden and associated with an acute febrile illness, in one instance pneumonia (de Lange, 1932), in another scarlet fever (Tsimenakes, 1902), and yet another 'following an infection' (Kinnier Wilson, 1933).

One of our two cases, Janet G., developed 'meningism' with tonsillitis. The compressioncone seen at autopsy appears to be consistent with the other evidence of increased intracranial tension. In the other case, Linda B., the neurological picture confirmed the impression that death was due to an intracranial disturbance rather than to the comparatively insignificant bronchopneumonia demonstrated post-mortem. The cerebrospinal fluid pressure was not measured in this latter case, in view of the unfortunate consequences attributed to this procedure in the former, but the autopsy finding of dry and flattened cerebral convolutions is evidence that intracranial pressure was raised.

If, in these two cases, death was due to a relatively sudden rise in intracranial tension, it is interesting to speculate on its possible cause. In the normal course of events, variation in the amount of cerebrospinal fluid in the cranium may compensate for a moderate increase in volume of the other intracranial contents; but in cases of megalencephaly it may well be that the margin of safety is too small for adaptation, so that any increase in quantity of cerebrospinal fluid or in brain bulk may lead to a fatal rise of intracranial pressure.

A relatively sudden increase of intracranial tension may result from various causes. In meningism an excess of dilute cerebrospinal fluid is formed, probably as a result of rapid dilution of the blood in acute infections and increased filtration through the choroid plexus from the relatively hypotonic plasma (Fremont-Smith and Merritt, 1937). In neither of our cases was there evidence of dilution of the cerebrospinal fluid, an essential feature of meningism, and only slight clinical evidence of the condition was found in case 1 , Janet G. An increased secretion of cerebrospinal fluid would not, therefore, appear to be the explanation of the raised intracranial tension in our cases, but it may explain some sudden deaths. Another possible explanation would be acute cerebral oedema. In neither case was there any evidence of this post mortem.

A further possibility is that the sudden rise in pressure results from an increased amount of blood within the cranium. Cairns (1939) has described acute swelling of the brain and distension of the capillaries and venous channels following inhalation of carbon dioxide during anaesthesia, and it is perhaps possible that in some cases bouts of apnoea or asphyxia may have a similar effect as a result of a rise in the carbon dioxide content of the blood.

The cause of death is not adequately explained by this mechanism in our first case; but our second case, Linda B., had pneumonia with bouts of apnoea and cyanosis, and at autopsy one of the striking features was the marked distension of all the intracranial venous sinuses. It is a possibility that the final explanation given above may apply in this instance.

\section{Summary}

Two cases of megalencephaly are reported, with clinical and pathological data.

In both cases death was associated with an acute infection, and it is suggested that death was due to a sudden increase in intracranial tension. Possible causes leading to this rise in intracranial pressure are discussed.

We thank Prof. C. Bruce Perry and Prof. T. F. Hewer for their very helpful suggestions, and members of the staff of the Bristol Children's Hospital for permission to publish these cases. We are especially grateful to Dr. R. M. Norman for his reports on the histology of the brains. For the photography we are indebted to Mr. S. A. Edwards.

\section{REFERENCES}

Brain, W. R. (1936). Proc. roy. Soc. Med., 30, 391. Cairns, H. (1939). Brit. J. Surg., 27, 275.

de Lange, C. (1932). Acta. Psych. Neurol., 7, 955.

Fletcher, H. Morley (1900). Trans. Pathol. Soc., 51, 230.

Fremont-Smith, F., and Merrit, H. H. (1937). The Cerebrospinal Fluid, London, p. 190.

Stewart, R. M. (1936-37). Proc. roy. Soc. Med., 30, 391. Tsimenakes, K. (1902). Cited Wilson, S. A. K. Neurology. London. 1940.

Wilson, S. A.K. (1933). J. Neurol.Psychopathol., 14,193. 


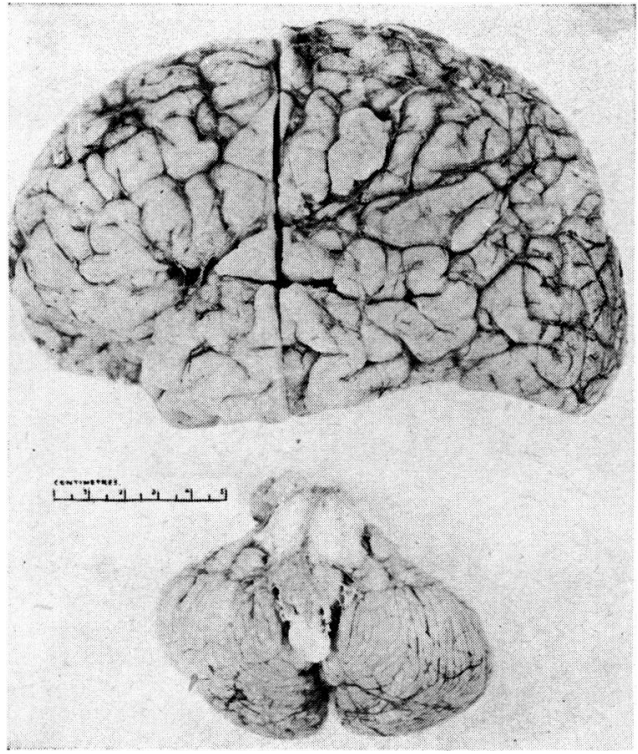

FIG. 1.-Brain of Janet G., age one year. Generalized enlargement. (Weight, 1,450 g.)

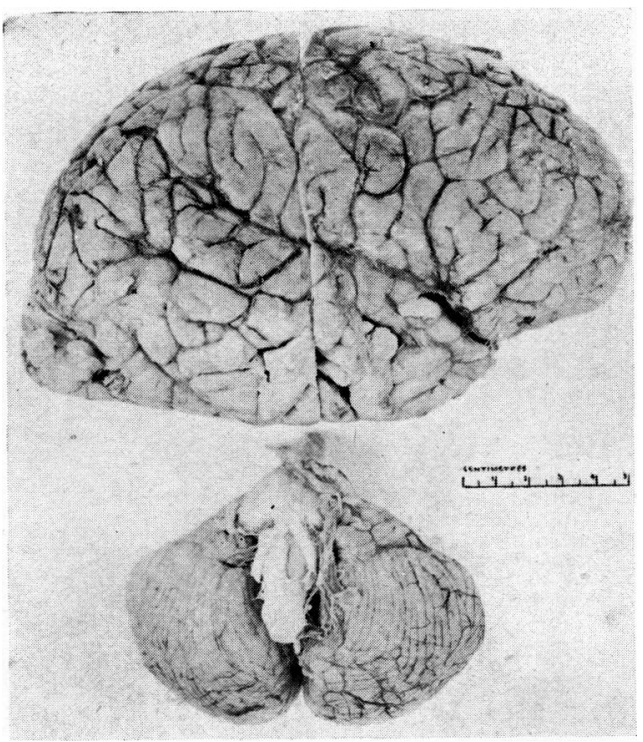

FIG. 2.-Brain of Linda B., age fifteen months. Generalized enlargement and flattening of convolutions. (Weight, 1,770 g.)

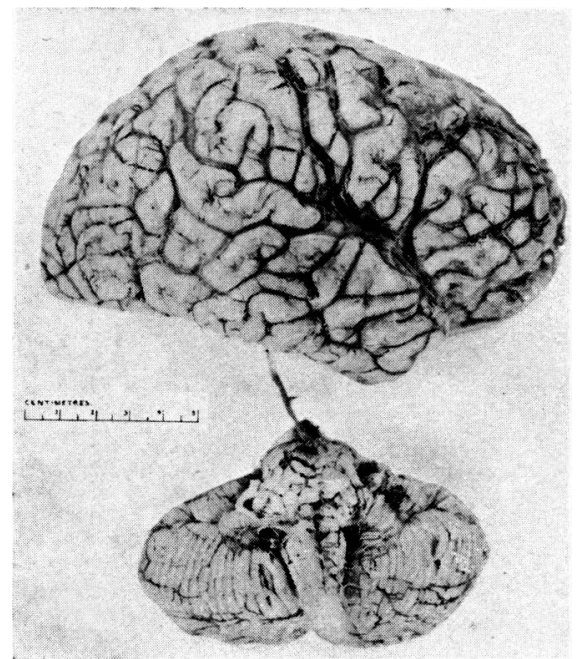

Fig. 3.-A normal adult female brain. (Weight, 1,300 g.) 